\title{
Diagnostic Value of Methylated Septin9 for Colorectal Cancer Detection
}

\author{
Li Xie ${ }^{1 \dagger}$, Xiyi Jiang ${ }^{2 \dagger}$, Qian $L^{1}{ }^{1}$, Zujun Sun ${ }^{3}$, Wenqiang Quan ${ }^{3,4}$, Yuping Duan ${ }^{1}$, Dong $L^{3 *}$ \\ and Tianhui Chen ${ }^{2 *}$
}

${ }^{1}$ Department of Clinical Laboratory, Shanghai First People's Hospital, BaoShan Branch, Shanghai, China, ${ }^{2}$ Group of Molecular Epidemiology \& Cancer Precision Prevention, Institute of Occupational Diseases, Zhejiang Academy of Medical Sciences, Hangzhou, China, ${ }^{3}$ Department of Clinical Laboratory, Shanghai Tongji Hospital, Tongji University School of Medicine, Shanghai, China, ${ }^{4}$ Department of Laboratory Medicine, Ninghai First People's Hospital, Ningbo, China

Background: Methylated Septin9 (mSEPT9) has been suggested as a reliable biomarker in colorectal cancer (CRC) detection. We aimed to determine the diagnostic value of mSEPT9 for CRC detection in Chinese patients. In addition, we compared the diagnostic efficacy of mSEPT9 to traditional screening method [fecal occult blood test (FOBT)] and two biomarkers [carcinoembryonic antigen (CEA) and carbohydrate antigen-199 (Ca-199)].

\section{OPEN ACCESS}

Edited by:

Dana Hashim,

International Agency For Research On Cancer (IARC), France

Reviewed by:

Sha Tao,

Fudan University, China

Zohreh Sanaat,

Tabriz University of Medical

Sciences, Iran

*Correspondence:

Dong Li

lidong@tongji.edu.cn;

Tianhui Chen

t.chen@zjams.com.cn

tThese authors have contributed equally to this work.

Specialty section: This article was submitted to Cancer Epidemiology and Prevention, a section of the journal

Frontiers in Oncology

Received: 24 April 2018 Accepted: 18 June 2018

Published: 02 July 2018

Citation:

Xie L, Jiang X, Li Q, Sun Z, Quan W, Duan Y, Li D and Chen T (2018) Diagnostic Value of Methylated Septing for Colorectal Cancer

Detection.

Front. Oncol. 8:247. doi: 10.3389/fonc.2018.00247
Methods: Overall 248 subjects including 123 patients with CRC and 125 controls were included. Plasma and fecal samples were collected for CEA, Ca-199, mSEPT9, and FOBT tests. Sensitivity and specificity were calculated to evaluate the diagnostic efficacy of each method; receiver operating characteristic $(\mathrm{ROC})$ curve was plotted for the assessment of diagnostic accuracy, and comparisons among FOBT, mSEPT9, and the combination were assessed through area under the ROC curve (AUC).

Results: ISEPT9 achieved overall sensitivity and specificity of $61.8 \%$ [95\% confidence interval (Cl): 53.0-69.9\%] and 89.6\% (83.0-93.8\%), respectively, with an AUC value of 0.757 (95\% Cl: 0.701-0.807), superior to FOBT [sensitivity: $61.4 \%$ (50.9-70.9\%); specificity: 70.3\% (59.1-79.5\%); AUC: 0.658 (0.578-0.723)], CEA [sensitivity: 35.0\% (27.1-43.7\%); specificity: 62.6\% (53.8-70.7\%); AUC: 0.485 (0.411-0.559)], and Ca-199 [sensitivity: 17.9\% (12.1-25.6\%); specificity: 55.7\% (48.9-64.1\%); AUC: 0.353 (0.2830.423)]. The combination of mSEPT9 and FOBT further improved sensitivity and AUC value of $84.1 \%(75.1-90.3 \%)$ and $0.807(0.752-0.863)$, respectively, while specificity was declined to $62.2 \%(50.8-72.4 \%)$.

Conclusion: $m S E P T 9$ demonstrated best diagnostic ability in CRC detection compared with FOBT, CEA, and Ca-199. The combination of mSEPT9 and FOBT further improved diagnostic sensitivity especially for early stage disease, which may provide a new approach for future CRC screening, though further investigations are warranted.

Keywords: colorectal cancer, biomarker, septin9, methylation, early detection

\section{INTRODUCTION}

Colorectal cancer (CRC) is one of the most common gastrointestinal malignancies across the globe. According to GLOBOCAN 2012, CRC was the third most common cancer and the fourth leading cause of cancer-related death worldwide, accounting for approximately 1,360,000 new cases and 694,000 deaths per year (1). In China, the incidence and mortality rate of CRC both rank fifth 
among all malignant cancers (2); its incidence and mortality rate shall continue to increase along with the development of social economy and residents' westernized lifestyle in China (2).

Screening has been shown to reduce CRC mortality $(3,4)$. Fecal occult blood test (FOBT) is widely used because of the low cost and non-invasiveness, whereas the sensitivity of FOBT alone for CRC screening is relatively low (commonly below 70\%) due to many confounders $(5,6)$. Colonoscopy is currently considered as the gold standard for CRC detection $(7,8)$. However, high cost, invasive procedure and relatively high risk of complications preclude its wide implement especially in some undeveloped regions (9). Moreover, carcinoembryonic antigen (CEA) and carbohydrate antigen-199 (Ca-199) are used as blood-based tumor biomarkers but show unsatisfactory performance (10). Therefore, CEA and Ca-199 are not recommended for CRC screening while can be used for monitoring response to surgical or systemic therapy (11). Given that the ineffective or invasive of the traditional screening methods, blood-based biomarkers with high sensitivity, specificity, and compliance for CRC screening is urgently warranted for early detection of CRC.

It is well known that CRC occurs due to the genetic and epigenetic alterations of intestinal epidermal cells, experiencing three stages: molecular change, cellular change, and tissue change (12). Thus, the determination of specific molecular markers targeting the related changes may be a promising method for detecting early CRC (13). Recently, DNA methylation-based biomarkers have become a hot spot in cancer research. While DNA methylation is essential for the regulation of gene expression and the maintenance of cellular identity, epigenetic changes through altered DNA methylation play an important role in tumorigenesis (14). DNA methylation mainly occurs at C-phosphate-G (CpG) sites, in which unmethylated $\mathrm{CpGs}$ existing in clusters are called $\mathrm{CpG}$ islands. Aberrant methylation of $\mathrm{CpG}$ islands in promoter regions of genes has been linked to epigenetic transcriptional silencing of tumor suppressor genes, which appears to be crucial in the early stages of CRC development (15).

Several DNA methylation-based biomarkers for CRC have been reported in previous studies, of which, methylated Septin 9 ( $m S E P T 9$ ) is considered as a promising one for detecting CRC (16). SEPT9, located at chromosome 17q25.3, is a conservative skeletal protein gene with GTPas activity, involving in cytokinesis and cytoskeletal organization $(17,18)$. SEPT9 is closely related to CRC carcinogenesis when the promoter region is hypermethylated and the transcription is compromised (19). The relationship between mSEPT9 and CRC makes it possible to be used as an informative tumor biomarker. Specifically, mSEPT9 DNA is released into the peripheral blood from necrotic and apoptotic cancer cells during CRC carcinogenesis; therefore, the risk of CRC can be determined by detecting the degree of DNA methylation of specific promoter region of SEPT9 in the peripheral blood (20). Up to now, several studies have evaluated the diagnostic value of $m S E P T 9$ in CRC detection, yet the diagnostic accuracy differs significantly between each study, in which the sensitivity and specificity varied from 36.6 to $95.6 \%$ and 77 to $98.9 \%$, respectively (20-24). Among them, few studies used Chinese CRC patients, with the sensitivity and specificity ranging from 69 to $88 \%$ and 87 to $98 \%$, respectively (25-28). Therefore, it remains to be determined whether $m S E P T 9$ is a reliable biomarker for CRC detection in Chinese population.

In this study, we aimed to determine the diagnostic value of mSEPT9 for blood-based CRC detection in a Chinese population. In addition, we compared the diagnostic efficacy of $m S E P T 9$ to traditional screening method (FOBT) and two blood-based tumor biomarkers (CEA and Ca-199), and in combinations among aforementioned biomarkers.

\section{MATERIALS AND METHODS}

\section{Study Subjects and Samples}

All samples were collected from Affiliated Hospital of Tongji University and Baoshan branch of Shanghai First People's Hospital. Subjects were recruited between October 1, 2016 and January 31, 2018. Only subjects who simultaneously performed CEA, Ca-199, and mSEPT9 examinations were enrolled; and among them who received chemotherapy/surgical intervention were also excluded. Ultimately, overall 248 subjects were included in this study, including 123 CRC patients and 125 controls (diagnosed without CRC). Demographic and clinical-pathological information of subjects including sex, age, pathological type, tumor stage, and metastasis status were collected. CRC cases were confirmed by pathological diagnosis. Tumor stages were defined according to TNM staging system of 7 th edition of the Cancer Staging Manual of American Joint Committee on Cancer (29).

This study was approved by the ethics committee of Affiliated Hospital of Tongji University and Baoshan branch of Shanghai First People's Hospital. Collection of samples and clinical-pathological information from subjects were undertaken with informed consent.

\section{SEPT9 Methylation Detection DNA Preparation and Bisulfite Conversion From Plasma Specimens}

For each sample, $3 \mathrm{~mL}$ blood was collected in an EDTA vacutainer tube (it was difficult to collect $10 \mathrm{~mL}$ blood, though $10 \mathrm{~mL}$ blood was initially requested for each subject). Each tube was immediately centrifuged at $1,350 \times g$ for $10 \mathrm{~min}$ at room temperature. Plasma was transferred to a $2-\mathrm{mL}$ tube without disturbing the pellet and stored at $-20^{\circ} \mathrm{C}$. The genomic DNA was extracted from $1 \mathrm{~mL}$ plasma using a Genomic DNA extraction Kit with magnetic beads from Tellgen Corporation, following the product protocol. Sample DNA was treated with bisulfite conversion reagents and purified by purification reagent, purchasing from Zymo Research (EZ DNA Methylation-Direct ${ }^{\mathrm{TM}}$ Kit) $(30,31)$. The unmethylated $\mathrm{C}$ bases in genomic DNA were modified to $\mathrm{U}$ bases by bisulfite, while the methylated $\mathrm{C}$ bases remained unchanged. Thus, the methylated and unmethylated $\mathrm{C}$ bases could be distinguished. For bisulfite conversion, we added $20 \mu \mathrm{L}$ DNA (concentration between 10 and $50 \mathrm{ng} / \mu \mathrm{L}$ ) to $130 \mu \mathrm{L}$ CT Conversion Reagent (fresh prepared following the product protocol) in a $200-\mu \mathrm{L}$ PCR tube and performed the conversion using a PCR program: predenaturation at $98^{\circ} \mathrm{C}$ for $8 \mathrm{~min}, \mathrm{CT}$ Conversion at $64^{\circ} \mathrm{C}$ for $3.5 \mathrm{~h}$, stored at $4^{\circ} \mathrm{C}$ up to $20 \mathrm{~h}$. We purified bis-DNA using Zymo-Spin ${ }^{\mathrm{TM}}$ IC Column. Purified bis-DNA was eluted in $20-\mu \mathrm{L}$ elution buffer 
(M-Elution Buffer) and used directly in methylation-specific real-time PCR (MSP) analysis.

\section{Methylation-Specific Real-Time PCR}

Methylated Septin9 and beta-actin (ACTB) as internal control were performed in the same reaction. MSP was used to preferentially detect the methylated form of SEPT9. Following the bisulfite modification, MSP was performed with primers designed to amplify the methylated sequences in a part of SEPT9-v2 promoter. The DNA was analyzed by the Methylated Human SEPT9 Gene Detection Kit (Tellgen Corporation) following the product protocol. The PCR mixtures contained $5 \mu \mathrm{L}$ modified DNA, $15 \mu \mathrm{L}$ PCR Mix including PCR reaction buffer, oligonucleotide primers, labeled probes and HotStart Taq DNA polymerase. The thermal cycling profile for PCR was set up as follows: pre-denaturation at $95^{\circ} \mathrm{C}$ for $10 \mathrm{~min}, 5 \mathrm{cycles}$ of denaturation for $15 \mathrm{~s}$ at $95^{\circ} \mathrm{C}$, annealing for $30 \mathrm{~s}$ at $60^{\circ} \mathrm{C}$, and $40 \mathrm{cycles}$ of denaturation for $15 \mathrm{~s}$ at $95^{\circ} \mathrm{C}$, annealing for $32 \mathrm{~s}$ at $56^{\circ} \mathrm{C}$. MSP was performed on the ABI7500 (Applied Biosystems).

\section{PCR Data Analysis}

PCR curves for the mSEPT9 and ACTB were generated. mSEPT9 was "detected" if the quantification cycle ("cycle threshold," $\mathrm{Ct}$ ) was less than 35 cycles. Plasma specimens were "not detected" if the $m S E P T 9 \mathrm{Ct}$ was not measurable or was $\geq 35$ cycles and the ACTB Ct $<35$ cycles. If ACTB was not detected, sample DNA was treated with bisulfite conversion and MSP.

\section{Fecal Occult Blood Test}

Colloidal gold immunochromatography with double antibody sandwich assay was used for the qualitative detection of fecal samples. A total of 8-mL fecal samples were detected using a WWT/ FA160-Auto analyzer (Wowente Biotechnology Co. Ltd., Sichuan, China), with a $200 \mathrm{ng} / \mathrm{mL}$ hemoglobin cutoff test positivity.

\section{Carcinoembryonic Antigen}

A total of 3-5 mL of venous blood was collected, and serum was isolated by centrifugation at 3,000 rpm for $15 \mathrm{~min}$. Electronic chemiluminescence immunoassay (ECLIA) Kit (Roche Diagnostics $\mathrm{GmbH}$ ) was used for CEA quantitative detection according to the manufacture's instruction. A cutoff CEA value of $5.2 \mathrm{ng} / \mathrm{mL}$ was considered positive. Serum samples were detected immediately.

\section{Carbohydrate Antigen-199}

A total of 3-5 mL of venous blood was collected, and serum was isolated by centrifugation at 3,000 rpm for $15 \mathrm{~min}$. ECLIA Kit (Roche Diagnostics $\mathrm{GmbH}$ ) was used for Ca-199 quantitative detection according to the manufacture's instruction. A cutoff Ca-199 value of $39.0 \mathrm{U} / \mathrm{mL}$ was considered positive. Serum samples were detected immediately.

\section{Statistical Analysis}

The differences of sex and age between case group and control group were analyzed using chi-square test and $t$-test, respectively. Detection results of $m S E P T 9$ were analyzed using positive rate and negative rate, and differences in detection rates among sex, age, and tumor location were analyzed using chi-square test or
Fisher's exact test. Sensitivity and specificity with $95 \%$ confidence intervals (CIs) were calculated to evaluate the diagnostic efficacy of each method (FOBT, CEA, Ca-199, mSEPT9, and the combination of FOBT and mSEPT9) in CRC detection. Meanwhile, diagnostic efficacies of methods of FOBT, mSEPT9, and the combinations in different tumor location, tumor stage, and metastasis status were evaluated and compared by sensitivity. Differences in sensitivity were analyzed using chi-square test or Fisher's exact test. Furthermore, the receiver operating characteristic (ROC) curve was used to evaluate the diagnostic accuracy, and the comparisons among methods of FOBT, $m S E P T 9$, and the combination were evaluated by the area under the ROC curve (AUC).

All statistical analyses were performed using the SAS statistical software, version 9.4 (SAS Institute Inc., Cary, NC, USA). Two-side $P$-value $<0.05$ was considered statistically significant.

\section{RESULTS}

\section{Demographic and Clinical Features of the Subjects}

Table 1 presents basic characteristics on subjects. Overall 248 subjects were enrolled in the study, including 139 men (56.0\%) and 109 women (44.0\%). The median age was 66 years and the majority (73.4\%) aged at least 60 years. Subjects in CRC case and control groups were 123 (74 men, 49 women, median age 66.07 years) and 125 (65 men, 60 women, median age 66.17 years), respectively. No significant differences in sex and age between the case group and control group were observed $(P>0.05)$. In the case group, 77 $(62.5 \%)$ and $46(37.5 \%)$ patients were diagnosed with colon cancer and rectum cancer, respectively. Within the colon cancer case group, tumor locations in sigmoid colon consisted of the largest proportion of $26.8 \%(33 / 123)$, compared with tumor locations in ileocecal junction (4.9\%), ascending colon (6.5\%), transverse colon (2.4\%), splenic flexure colon (1.6\%), descending colon $(0.8 \%)$, and colon unspecified (19.5\%). CRC patients in stage I, stage II, stage III, and stage IV were 5 (4.9\%), 36 (35.0\%), 58 (56.3\%), and 4 (3.8\%), respectively. In addition, CRC patients with regional lymph node metastasis, distant metastasis, and vascular and neural infiltration were 45,6 , and 25 , respectively. In the control group, patients with bowel diseases consisted of the largest proportion of $40.8 \%$ (51/125), compared with the patients with malignancies in other systems (8.8\%), other diseases (35.2\%), and healthy control (15.2\%).

\section{Detection Results of $\mathrm{mSEPT9}$}

Overall 89 subjects were detected with $m S E P T 9$ positive, including 55 men (39.6\%) and 34 women (31.2\%); no sex difference was observed (Table 2 ). Older subjects (aged $\geq 60$ years) had significantly higher positive rates of $m S E P T 9$, compared with younger subjects ( 40.1 vs. $24.2 \%$ ). CRC case group had significantly higher positive rates of $m S E P T 9$, compared with the control group (61.8 vs. $10.4 \%)$. Within the CRC case group, patients with colon cancer had higher positive rates of $m$ SEPT9, compared with rectum cancer (67.5 vs. $52.2 \%)$, though the difference did not reach statistical significance $(P=0.09)$. In the control group, patients with malignancies in other systems had significantly higher positive rates of $\operatorname{LSEPT9}(36.4 \%)$, 
TABLE 1 | Demographic and clinical features of the subjects.

\begin{tabular}{|c|c|c|c|}
\hline & Total $(n, \%)$ & $\operatorname{CRC}(n, \%)$ & Control (n, \%) \\
\hline $\operatorname{Sex}^{a}$ & 248 & 123 & 125 \\
\hline Male & $139(56.0)$ & $74(60.2)$ & 65 (52.0) \\
\hline Female & $109(44.0)$ & 49 (39.8) & $60(48.0)$ \\
\hline \multicolumn{4}{|l|}{ Age (years) ${ }^{b}$} \\
\hline$<60$ & 66 (26.6) & $31(25.2)$ & $35(28.0)$ \\
\hline$\geq 60$ & $182(73.4)$ & $92(74.8)$ & $90(72.0)$ \\
\hline Median age (years) & 66.12 & 66.07 & 66.17 \\
\hline \multicolumn{4}{|l|}{ Tumor location } \\
\hline Colon & - & 77 (62.5) & - \\
\hline lleocecal junction & - & $6(4.9)$ & - \\
\hline Ascending colon & - & $8(6.5)$ & - \\
\hline Transverse colon & - & $3(2.4)$ & - \\
\hline Splenic flexure colon & - & $2(1.6)$ & - \\
\hline Descending colon & - & $1(0.8)$ & - \\
\hline Sigmoid colon & - & $33(26.8)$ & - \\
\hline Colon, unspecified & - & $24(19.5)$ & - \\
\hline Rectum & - & $46(37.5)$ & - \\
\hline \multicolumn{4}{|l|}{ Non-CRC } \\
\hline Bowel diseases & & - & $51(40.8)$ \\
\hline Malignancies in other systems & & - & $11(8.8)$ \\
\hline Other diseases & & - & $44(35.2)$ \\
\hline Healthy control & & - & $19(15.2)$ \\
\hline \multicolumn{4}{|l|}{ Tumor stage ${ }^{c}$} \\
\hline Stage I & & $5(4.9)$ & - \\
\hline Stage II & & $36(35.0)$ & - \\
\hline Stage III & & $58(56.3)$ & - \\
\hline Stage IV & & $4(3.8)$ & - \\
\hline \multicolumn{4}{|l|}{ Regional lymph node metastasis ${ }^{c}$} \\
\hline Yes & & $45(43.7)$ & - \\
\hline No & & $58(56.3)$ & - \\
\hline \multicolumn{4}{|l|}{ Distant metastasis ${ }^{c}$} \\
\hline Yes & & $6(5.8)$ & - \\
\hline No & & $97(94.2)$ & - \\
\hline \multicolumn{4}{|l|}{ Vascular and neural infiltration ${ }^{c}$} \\
\hline Yes & & $25(24.3)$ & - \\
\hline No & & $78(75.7)$ & - \\
\hline
\end{tabular}

CRC, colorectal cancer.

Bowel diseases including bowel polyp, bowel obstruction, and enteritis.

Malignancies in other systems including stomach cancer, lung cancer, liver cancer,

pancreatic cancer, and breast cancer.

${ }^{a} P=0.195$.

${ }^{b} P=0.956$.

Information was missing among 20 subjects ( $n=103)$.

compared with those with bowel diseases (9.8\%) and with other diseases (9.1\%). Notably, none of healthy control was detected with $m$ SEPT9 positive.

\section{Diagnostic Efficacy of Each Method}

As is shown in Table 3, mSEPT9 showed the highest diagnostic ability with a sensitivity of $61.8 \%$ (95\% CI: 53.0-69.9\%), compared with FOBT [61.4\% (50.9-70.9\%)], CEA [35.0\% (27.1-43.7\%)], and Ca-199 [17.9\% (12.1-25.6\%)] $(P<0.05)$. Meanwhile, $m$ SEPT9 also had the highest specificity [89.6\% (83.0-93.8\%)], compared with FOBT [70.3\% (59.1-79.5\%)], CEA [62.6\% (53.8-70.7\%)], and Ca-199 [55.7\% (48.9-64.1\%)] $(P<0.05)$. Notably, the combination of $m S E P T 9$ with FOBT further improved sensitivity, reaching $84.1 \%(75.1-90.3 \%)$, though the specificity declined [62.2\% (50.8-72.4\%)].

Table 4 presents further stratifications by tumor location. mSEPT9 had higher sensitivity for colon cancer compared with
TABLE 2 | Detection results of mSEPT9 $(n=248)$.

\begin{tabular}{|c|c|c|c|c|c|c|c|}
\hline & \multicolumn{4}{|c|}{ Positive (n, \%) } & \multicolumn{2}{|c|}{ Negative $(n, \%)$} & b) $P$-value \\
\hline \multicolumn{2}{|l|}{ Sex } & \multicolumn{3}{|c|}{89} & \multicolumn{2}{|r|}{159} & \\
\hline \multicolumn{2}{|l|}{ Male } & \multicolumn{3}{|c|}{55 (39.6) } & \multicolumn{2}{|c|}{$84(60.4)$} & 0.172 \\
\hline \multicolumn{2}{|l|}{ Female } & \multicolumn{3}{|c|}{$34(31.2)$} & \multicolumn{2}{|c|}{75 (68.8) } & \\
\hline \multicolumn{8}{|l|}{ Age (years) } \\
\hline$<60$ & & \multicolumn{3}{|c|}{$16(24.2)$} & \multicolumn{2}{|c|}{$50(75.8)$} & $0.021^{*}$ \\
\hline$\geq 60$ & & \multicolumn{3}{|c|}{$73(40.1)$} & \multicolumn{2}{|c|}{109 (59.9) } & \\
\hline \multicolumn{8}{|l|}{ Tumor location ${ }^{c}$} \\
\hline \multicolumn{2}{|l|}{ Colon } & \multicolumn{3}{|c|}{$52(67.5)$} & \multicolumn{2}{|c|}{$25(32.5)$} & $0.090^{\mathrm{a}}$ \\
\hline \multicolumn{2}{|l|}{ Rectum } & \multicolumn{3}{|c|}{$24(52.2)$} & \multicolumn{2}{|c|}{$22(47.8)$} & $<0.0001^{\mathrm{b}}$ \\
\hline Total & & \multicolumn{3}{|c|}{$76(61.8)$} & \multicolumn{2}{|c|}{$47(38.2)$} & \\
\hline \multicolumn{8}{|l|}{ Non-CRC } \\
\hline \multicolumn{2}{|l|}{ Bowel diseases } & \multicolumn{3}{|c|}{$5(9.8)$} & \multicolumn{2}{|c|}{$46(90.2)$} & $0.030^{*}$ \\
\hline \multicolumn{2}{|c|}{ Malignancies in other sys } & \multicolumn{3}{|c|}{$4(36.4)$} & \multicolumn{2}{|c|}{7 (63.6) } & \\
\hline \multicolumn{2}{|l|}{ Other diseases } & & $4(9.1)$ & & 40 & $(90.9)$ & \\
\hline Healthy control & & & - & & 19 & $(100.0)$ & \\
\hline Total & & & $13(10.4)$ & & 112 & (89.6) & \\
\hline $\begin{array}{l}\text { mSEPT9, methylated } \\
{ }^{*} P<0.05 \text { indicates } s \\
{ }^{a} \text { Comparation betwe } \\
{ }^{b} \text { Comparation betwe } \\
{ }^{c} n=123 . \\
{ }^{d} n=125 .\end{array}$ & $\begin{array}{l}\text { d Septing } \\
\text { statistical } \\
\text { en patier } \\
\text { een subje }\end{array}$ & $\begin{array}{l}\text { 9; CRC, color } \\
\text { lly significant. } \\
\text { ints of colon c } \\
\text { ects in CRC gr }\end{array}$ & $\begin{array}{l}\text { rectal can } \\
\text { cancer an } \\
\text { group and }\end{array}$ & $\begin{array}{l}\text { ncer. } \\
\text { hd rec } \\
\text { d non- }\end{array}$ & $\begin{array}{l}\text { ctum can } \\
\text {-CRC grc }\end{array}$ & $\begin{array}{l}\text { ncer. } \\
\text { roup. }\end{array}$ & \\
\hline & Sensi & itivity (\%) & Speci & ificity & ty (\%) & & AUC \\
\hline & Value & $95 \% \mathrm{Cl}$ & Value & & $\%$ Cl & Value & $95 \% \mathrm{Cl}$ \\
\hline $\mathrm{FOBT}^{\mathrm{a}}$ & 61.4 & $50.9-70.9$ & 70.3 & 59.1 & $1-79.5$ & 0.6580 & $0.578-0.723$ \\
\hline CEA & 35.0 & $27.1-43.7$ & 62.6 & 53.8 & $8-70.7$ & 0.485 & $0.411-0.559$ \\
\hline Ca-199 & 17.9 & $12.1-25.6$ & 55.7 & 48.9 & $9-64.1$ & 0.3530 & $0.283-0.423$ \\
\hline mSEPT9 & 61.8 & $53.0-69.9$ & 89.6 & 83.0 & 0-93.8 & $0.757 \quad 0$ & $0.701-0.807$ \\
\hline $\mathrm{FOBT}^{+} m$ SEPT9 ${ }^{\mathrm{a}}$ & 84.1 & $75.1-90.3$ & 62.2 & 50.8 & $8-72.4$ & $0.807 \quad 0$ & $0.740-0.875$ \\
\hline
\end{tabular}

TABLE 4 | Detection sensitivity of fecal occult blood test (FOBT), MSEPT9, and the combination for the stratification by tumor location (\%).

\begin{tabular}{lccc}
\hline & FOBT $^{\mathbf{a}}$ & mSEPT9 & FOBT + ISEPT9 $^{\mathbf{a}}$ \\
\hline Colon $(n=77)$ & 57.8 & 67.5 & 86.0 \\
Right colon $(n=14)$ & 63.6 & 71.4 & 90.9 \\
Ileocecal junction $(n=6)$ & 50.0 & 66.7 & 100.0 \\
Ascending colon $(n=8)$ & 71.4 & 75.0 & 85.7 \\
Left colon $(n=36)$ & 59.3 & 66.7 & 92.6 \\
Splenic flexure and descending & 50.0 & 0 & 50.0 \\
colon $(n=3)$ & & & \\
Sigmoid colon $(n=33)$ & 60.0 & 72.7 & 96.0 \\
Transverse colon $(n=3)$ & 66.7 & 66.7 & 66.7 \\
Colon, unspecified $(n=24)$ & 50.0 & 66.7 & 75.0 \\
Rectum $(n=46)$ & 67.7 & 52.2 & 80.7
\end{tabular}

${ }^{a}$ FOBT information was missing among 20 patients in group of colon cancer, including 2 in ileocecal junction, 1 in ascending colon, 1 in descending colon, 8 in sigmoid colon, and 8 in unspecified colon, and FOBT information was missing among 15 patients in group of rectum cancer.

rectum cancer (67.5 vs. $52.2 \% ; P>0.05)$. $m$ SEPT9 had higher sensitivity for cancer at right colon compared with cancer at left colon (71.4 vs. $66.7 \%$; $P>0.05$ ); for right colon, $m S E P T 9$ had 
higher sensitivity for ascending colon cancer, compared with ileocecal junction cancer ( 75.0 vs. $66.7 \% ; P>0.05$ ). For colon cancer, $m S E P T 9$ achieved higher sensitivity, compared with FOBT for overall (67.5 vs. 57.8\%; $P>0.05$ ), right colon $(71.4$ vs. $63.6 \% ; P>0.05)$, and left colon (66.7 vs. $59.3 \% ; P>0.05)$, also similar performance for tumor locations of ileocecal junction, ascending colon, sigmoid colon, and unspecified colon $(P>0.05)$ (Table 4). However, for rectum cancer, $m S E P T 9$ had lower sensitivity, compared with FOBT (55.2 vs. 67.7\%; $P>0.05)$. Notably, when combined by $m S E P T 9$ with FOBT, the sensitivity further improved, reaching $86.0 \%$ for colon and $80.7 \%$ for rectum. Actually, the sensitivity improved for all stratifications except for transverse colon (remaining the same $66.7 \%)$. For instance, the sensitivity reached $90.9 \%$ for right colon (100\% for ileocecal junction), and $92.6 \%$ for left colon (96.0\% for sigmoid colon).

Table 5 presents the stratifications by tumor stage and tumor metastasis. mSEPT9 had slightly lower sensitivity compared with FOBT for overall stage (59.2 vs. $63.6 \% ; P>0.05$ ), and for stages I-III, except for stage IV, but the number was very small (4 cases) for stage IV. mSEPT9 had higher sensitivity compared with FOBT for patients with regional lymph node metastasis (60.0 vs. $54.3 \% ; P>0.05$ ), for patients with distant metastasis ( 83.3 vs. $33.3 \%$; $P>0.05$ ), and for patients with vascular and neural infiltration ( 56 vs. $52.9 \% ; P>0.05$ ). Interestingly, $m S E P T 9$ had higher sensitivity for patients with distant metastasis, compared with those without ( 83.3 vs. $57.7 \% ; P>0.05$ ), though the number was small for patients with distant metastasis $(n=6)$. When combined by mSEPT9 with FOBT, the sensitivity significantly improved, reaching $85.7 \%$ for overall, $100.0 \%$ for stage I, $82.6 \%$ for stage II, $88.9 \%$ for stage III, and $50.0 \%$ for stage IV. Besides, when combined by $m S E P T 9$ with FOBT, the sensitivity improved for all subgroups, reaching $85.7 \%$ for patients with regional lymph node metastasis, $83.3 \%$ for patients with distant

TABLE 5 | Detection sensitivity of fecal occult blood test (FOBT), mSEPT9, and the combination for the stratification by tumor stage and metastasis (\%).

\begin{tabular}{lccc}
\hline & FOBT $^{\mathbf{a}}$ & mSEPT9 & FOBT + mSEPT9 $^{\mathbf{a}}$ \\
\hline Stage $(n=103)$ & 63.6 & 59.2 & 85.7 \\
Stage I $(n=5)$ & 80.0 & 60.0 & 100.0 \\
Stage II $(n=36)$ & 56.5 & 52.8 & 82.6 \\
Stage III $(n=58)$ & 68.9 & 63.8 & 88.9 \\
Stage IV $(n=4)$ & 25.0 & 50.0 & 50.0 \\
\hline Regional Iymph node metastasis $(n=103)$ & & \\
Yes $(n=45)$ & 54.3 & 60.0 & 85.7 \\
No $(n=58)$ & 71.4 & 58.6 & 85.7 \\
\hline Distant metastasis $(n=103)$ & & & \\
Yes $(n=6)$ & 33.3 & 83.3 & 83.3 \\
No $(n=97)$ & 66.2 & 57.7 & 85.9 \\
\hline Vascular and neural infiltration $(n=103)$ & & \\
Yes $(n=25)$ & 52.9 & 56.0 & 82.4 \\
No $(n=78)$ & 66.7 & 60.3 & 86.7 \\
\hline
\end{tabular}

${ }^{a}$ FOBT information was missing among 26 patients, including 13 in group of stage II and 13 in stage III; FOBT information was missing among 10 and 8 patients in groups of regional lymph node metastasis (Yes) and vascular and neural infiltration (Yes), respectively, and FOBT information was missing among 16, 26, and 18 patients in groups of regional lymph node metastasis (No), distant metastasis (No), and vascular and neural infiltration (No), respectively. metastasis, and $82.4 \%$ for patients with vascular and neural infiltration.

\section{ROC Curve Analysis of FOBT, mSEPT9, and the Combination}

As is shown in Table 3 and Figure 1, mSEPT9 achieved the highest AUC value of 0.757 (95\% CI: 0.701-0.807), comparing to FOBT [0.658 (0.578-0.723)], CEA [0.485 (0.411-0.559)], and Ca-199 [0.353 (0.283-0.423)]. Besides, the combination of $m S E P T 9$ with FOBT further improved the AUC value, reaching 0.807 (0.752-0.863).

\section{DISCUSSION}

In this study, we evaluated the diagnostic value of mSEPT9 for blood-based CRC detection in Chinese patients, compared with traditional screening method (FOBT) and two blood-based tumor biomarkers (CEA and Ca-199). We found mSEPT9 achieved overall sensitivity of $61.8 \%$ (53.0-69.9\%) and specificity of $89.6 \%$ (83.0-93.8\%), with an AUC value of 0.757 (0.701-0.807), superior to FOBT [sensitivity: $61.4 \%$ (50.9-70.9\%); specificity: $70.3 \%$ (59.1-79.5\%); AUC: 0.658 (0.578-0.723)], CEA [sensitivity: 35.0\% (27.1-43.7\%); specificity: 62.6\% (53.8-70.7\%); AUC: 0.485 (0.411-0.559)], and Ca-199 [sensitivity: 17.9\% (12.1-25.6\%); specificity: $55.7 \%$ (48.9-64.1\%); AUC: 0.353 (0.283-0.423)]. The combination of $m S E P T 9$ with FOBT further improved sensitivity $[84.1 \%(75.1-90.3 \%)]$ and AUC value $[0.807(0.752-0.863)]$, though the specificity declined [62.2\% (50.8-72.4\%)].

Colorectal cancer is a common gastrointestinal malignancy with poor prognosis and high mortality rate, for which screening and early detection are crucial especially for high-risk population. Investigations on blood-based biomarkers for early detection of CRC are highly warranted because stool-based tests are not convenient. Our results indicated a poor performance of CEA and Ca-199 for detecting CRC, suggesting that they shall not be recommended for CRC screening, in line with previous studies $(10,11)$. Apart from the traditional biomarkers, growing evidence has shown a significant association between mSEPT9 and CRC pathogenesis, indicating the potential role of $m S E P T 9$ in CRC detection (32). mSEPT9 is a promising biomarker in CRC detection, with the advantage of convenience and non-invasiveness. Nevertheless, diagnostic sensitivity of mSEPT9 in CRC detection varied among published literatures. For European and American population, the sensitivity ranged from 58 to $95.6 \%(20,22-24$, $33-35)$; the sensitivity varied from 69 to $88 \%$ in Asia population, which was higher compared with our results (25-27). Yet, Lee et al. (21) found a very low sensitivity of $m$ SEPT9 (36.6\%) among Korean population. Interestingly, we found $m S E P T 9$ can be detected both in men and women for each age group and non-CRC diseases, with a higher positive rate among men and older subjects (aged $\geq 60$ years). Therefore, differences in sensitivity may be partly attributed to the sample heterogeneity caused by demographic characteristics (sex, race, and age), lifestyles (smoking and alcohol consumption), comorbidities, or other environmental exposure factors (36).

Methylated Septin9 can be detected in tumor locations of colon and rectum. mSEPT9 had higher sensitivity for colon 


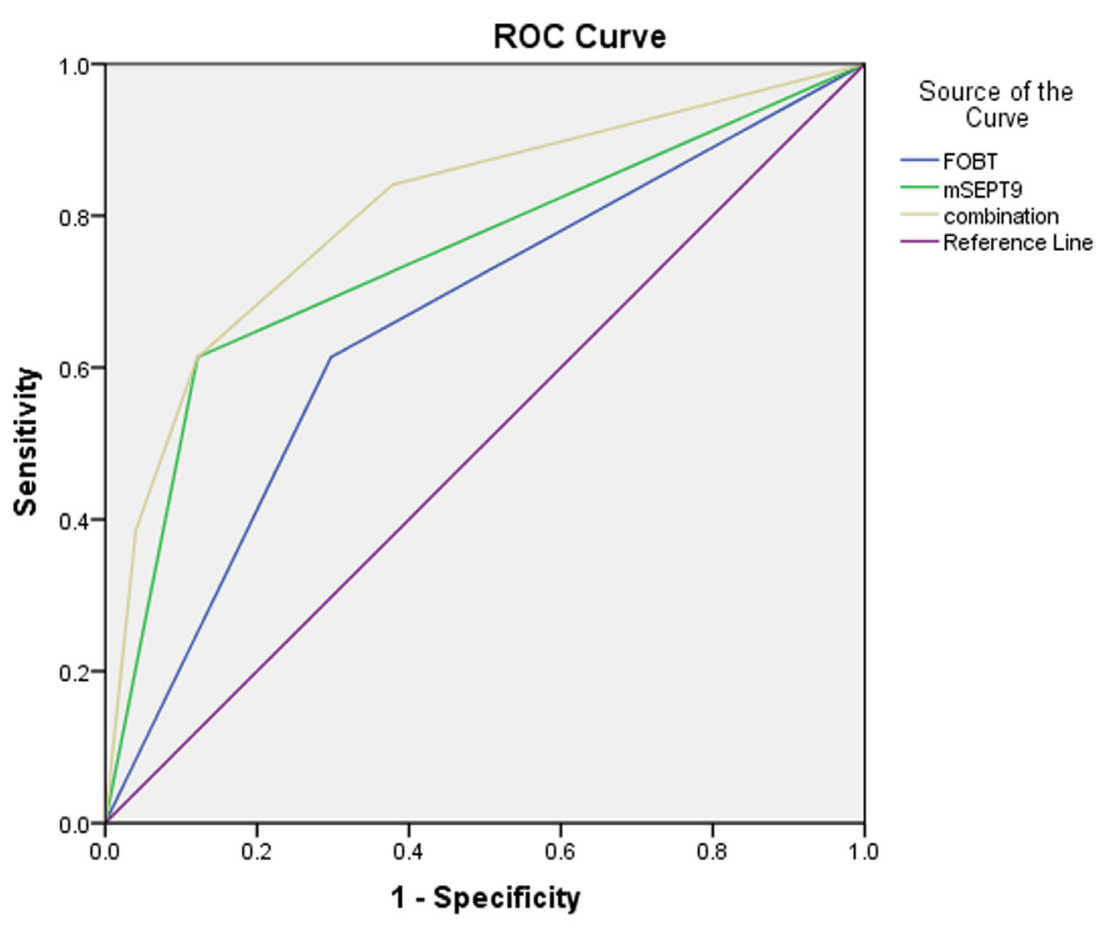

Diagonal segments are produced by ties.

FIGURE 1 | Receiver operating characteristic (ROC) curve of fecal occult blood test (FOBT), methylated Septin9 (mSEPT9), and the combination.

cancer compared with rectum cancer (67.5 vs. $52.2 \%$ ), in line with the investigation conducted by Li et al. (37). Besides, a higher sensitivity was found for cancer at right colon compared with cancer at left colon (71.4 vs. 66.7\%), which was similar to the findings from Li et al. (37). The reason may be partly attributed to different carcinogenesis for colon and rectum cancers.

We found mSEPT9 can be detected among all stages and the sensitivity reached highest (63.8\%) for stage III disease, consisted with the study by Jin et al. (38) showing a higher sensitivity for patients in stage III and stage IV and with the study by Ørntoft et al. (39) showing a higher sensitivity for patients with stage IIIV diseases. In addition, we found higher sensitivity for patients with advanced stage, such as metastasis (regional lymph node metastasis/distant metastasis). Our findings demonstrate that CRC patients with advanced stage are more easily detected by mSEPT9 compared with those with early stage.

We found the combination of $m$ SEPT9 with FOBT further improved the overall sensitivity [84.1\% (75.1-90.3\%)] and AUC value $[0.807(0.752-0.863)]$, in line with the study by Johnson et al. (40) showing the combination of mSEPT9 and FOBT further improved sensitivity (88.7\%) and with the study by Wu et al. (41) reporting of $94.4 \%$ sensitivity for the combination. For the stratification by tumor location, we found the combination further improved the sensitivity both for colon and rectum. Notably, we found $100.0 \%$ sensitivity for tumor location of ileocecal junction and for CRC patients in stage I. However, the number was small ( $n=6$ for patients with ileocecal junction cancer and $n=5$ for patients in stage I), further investigations with large number are highly warranted. Furthermore, the combination also improved the sensitivity for patients with advanced stage (with metastasis and infiltration). Taken together, our findings suggest that mSEPT9 could be a complementary tool (in addition to FOBT) for CRC detection.

This study has several strengths and limitations. First, CRC cases were confirmed by pathological diagnosis, which guarantee the diagnostic accuracy. Second, diagnostic efficacy of each method in different groups of tumor location, tumor stage, and metastasis were evaluated and compared, which could provide guidance for clinical application in the future. Major limitation concerns sample size. Small number for the stratifications might lead to an over-evaluated diagnostic value especially for some subgroups of tumor location. For instance, patients with rectum caner only accounted for a small proportion. Thus, further investigations with large number are warranted. Second, small volume $(3 \mathrm{~mL})$ of plasma samples were used for only one time detection, which was smaller compared with previous studies (both for the blood volume and detection number). Therefore, the results especially the sensitivity might be influenced. Third, while 35\% (86/248) subjects had missing value for fecal test results, the missing value was randomly distributed among case group but not among all subjects. Thus, the results might be biased. Furthermore, this study focuses on the diagnostic value of $m S E P T 9$ in CRC detection, yet the association between $m S E P T 9$ and prognosis of CRC patients is unknown, thus further study is needed. 
In conclusion, we found $m$ SEPT9 achieved overall sensitivity of $61.8 \%$ (53.0-69.9\%), specificity of $89.6 \%$ (83.0-93.8\%), and AUC value of 0.757 (0.701-0.807), superior to FOBT, CEA, and Ca-199. The combination of mSEPT9 with FOBT further improved sensitivity, though the specificity decreased. Therefore, further investigations on the diagnostic performance of the fecal test by different cutoff values are highly warranted. Along with the advantage of convenience and non-invasiveness, mSEPT9 is a promising biomarker in CRC detection. It could be expected that a further colonoscopy targeting the high-risk population ( $m$ SEPT9 positive) could improve the confirmation ability of CRC diagnosis, which avoids the repeated screening and reduces the invasive procedures. Combined use of mSEPT9 with traditional method of FOBT could improve the diagnostic sensitivity especially among CRC patients in early stage, which may provide a new approach for future CRC screening, while further investigations with large sample size are highly warranted.

\section{ETHICS STATEMENT}

This study was approved by the ethics committee of Affiliated Hospital of Tongji University and Baoshan branch of Shanghai First People's Hospital. Collection of samples and clinicalpathological information from subjects were undertaken with informed consent.

\section{REFERENCES}

1. Ferlay J, Soerjomataram I, Dikshit R, Eser S, Mathers C, Rebelo M, et al. Cancer incidence and mortality worldwide: sources, methods and major patterns in GLOBOCAN 2012. Int J Cancer (2015) 136(5):E359-86. doi:10.1002/ ijc. 29210

2. Chen W, Zheng R, Baade PD, Zhang S, Zeng H, Bray F, et al. Cancer statistics in China, 2015. CA Cancer J Clin (2016) 66(2):115-32. doi:10.3322/caac.21338

3. Singhal S, Changela K, Basi P, Mathur S, Reddy S, Momeni M, et al. Prescreening with FOBT improves yield and is cost-effective in colorectal screening in the elderly. ISRN Gastroenterol (2014) 2014(9):179291. doi:10.1155/2014/179291

4. Shroff J, Thosani N, Batra S, Singh H, Guha S. Reduced incidence and mortality from colorectal cancer with flexible-sigmoidoscopy screening: a meta-analysis. World J Gastroenterol (2014) 20(48):18466-76. doi:10.3748/ wjg.v20.i48.18466

5. Morikawa T, Kato J, Yamaji Y, Wada R, Mitsushima T, Shiratori Y. A comparison of the immunochemical fecal occult blood test and total colonoscopy in the asymptomatic population. Gastroenterology (2005) 129(2):422-8. doi:10.1053/j.gastro.2005.05.056

6. Yan S, Liu Z, Yu S, Bao Y. Diagnostic value of methylated septin9 for colorectal cancer screening: a meta-analysis. Med Sci Monit (2016) 22:3409-18. doi:10.12659/MSM.900590

7. Schoen RE, Pinsky PF, Weissfeld JL, Yokochi LA, Church T, Laiyemo AO, et al. Colorectal-cancer incidence and mortality with screening flexible sigmoidoscopy. New Engl J Med (2012) 366(25):2345-57. doi:10.1056/NEJMoa1114635

8. Uraoka T, Hosoe N, Yahagi N. Colonoscopy: is it as effective as an advanced diagnostic tool for colorectal cancer screening? Expert Rev Gastroenterol Hepatol (2015) 9(2):129-32. doi:10.1586/17474124.2015.960397

9. Inadomi JM, Vijan S, Janz NK, Fagerlin A, Thomas JP, Mun YVLR, et al. Adherence to colorectal cancer screening. Arch Intern Med (2012) 172(7):57582. doi:10.1001/archinternmed.2012.332

10. Wild N, Andres H, Rollinger W, Krause F, Dilba P, Tacke M, et al. A combination of serum markers for the early detection of colorectal cancer. Clin Cancer Res (2010) 16(24):6111-21. doi:10.1158/1078-0432.CCR-10-0119

11. Locker GY, Hamilton S, Harris J, Jessup JM, Kemeny N, Macdonald JS, et al. ASCO 2006 update of recommendations for the use of tumor markers in

\section{AUTHOR CONTRIBUTIONS}

LX and DL were responsible for the study concept and design. TC and DL obtained funding. LX and DL acquired data. LX and $\mathrm{XJ}$ analyzed and interpreted data. LX, XJ, and TC drafted the manuscript, and all authors revised it for important intellectual content. DL and TC are the guarantors of this work.

\section{FUNDING}

This work was supported by grants from National Key R\&D Program of China [grant number: 2017YFC0908200], key project of science and technologies of Shanghai [grant number: PKJ2014-S28], The Three-year Planning for Strengthening the Construction of the Public Health System in Shanghai (2015-2017) [grant number: 15GWZK0301], Key Research-Development Program of Zhejiang Province [grant number: 2017C03013], Joint Key Program of Zhejiang Province-Ministry of Health [grant number: WKJ-ZJ-1714], Natural Science Foundation of Zhejiang Province (No. LY16H070001), and Qianjiang Talents Fund of Zhejiang Province [grant number: QJD1602026]. The study sponsors were not involved in the study design, data collection, data analysis, interpretation of results, writing of the manuscript, and in the decision to submit the manuscript for publication.

gastrointestinal cancer. J Clin Oncol (2006) 24(33):5313-27. doi:10.1200/ JCO.2006.08.2644

12. Tariq K, Ghias K. Colorectal cancer carcinogenesis: a review of mechanisms. CancerBiolMed(2016) 13(1):120-35.doi:10.20892/j.issn.2095-3941.2015.0103

13. Bosch LJW, Carvalho B, Fijneman RJA, Jimenez CR, Pinedo HM, Engeld M, et al. Molecular tests for colorectal cancer screening. Clin Colorectal Cancer (2011) 10(1):8-23. doi:10.3816/CCC.2011.n.002

14. Cedar H, Bergman Y. Programming of DNA methylation patterns. Annu Rev Biochem (2012) 81(1):97-117. doi:10.1146/annurev-biochem-052610-091920

15. Lao VV, Grady WM. Epigenetics and colorectal cancer. Nat Rev Gastroenterol Hepatol (2011) 8(12):686-700. doi:10.1038/nrgastro.2011.173

16. Summers T, Langan RC, Nissan A, Brücher BRLD, Bilchik AJ, Protic M, et al. Serum-based DNA methylation biomarkers in colorectal cancer: potential for screening and early detection. J Cancer (2013) 4(3):210-6. doi:10.7150/ jca.5839

17. Mcilhatton MA, Burrows JF, Donaghy PG, Chanduloy S, Johnston PG, Russell SE. Genomic organization, complex splicing pattern and expression of a human septin gene on chromosome 17q25.3. Oncogene (2001) 20(41):5930-9. doi:10.1038/sj.onc. 1204752

18. Hall P, Russell S. The pathobiology of the septin gene family. J Pathol (2004) 204(4):489-505. doi:10.1002/path.1654

19. Jones PA, Baylin SB. The epigenomics of cancer. Cell (2007) 128(4):683-92. doi:10.1016/j.cell.2007.01.029

20. Tóth K, Sipos F, Kalmár A, Patai AV, Wichmann B, Stoehr R, et al. Detection of methylated SEPT9 in plasma is a reliable screening method for both left- and right-sided colon cancers. PLoS One (2012) 7(9):e46000. doi:10.1371/journal. pone. 0046000

21. Lee HS, Hwang SM, Kim TS, Kim DW, Park D, Kang SB, et al. Circulating methylated septin 9 nucleic acid in the plasma of patients with gastrointestinal cancer in the stomach and colon. Transl Oncol (2013) 6(3):290-6. doi:10.1593/ tlo. 13118

22. Warren JD, Xiong W, Bunker AM, Vaughn CP, Furtado LV, Roberts WL, et al. Septin 9 methylated DNA is a sensitive and specific blood test for colorectal cancer. BMC Med (2011) 9(1):133. doi:10.1186/1741-7015-9-133

23. Ahlquist DA, Taylor WR, Mahoney DW, Zou H, Domanico M, Thibodeau SN, et al. The stool DNA test is more accurate than the plasma septin 9 test in 
detecting colorectal neoplasia. Clin Gastroenterol Hepatol (2012) 10(3):272. doi:10.1016/j.cgh.2011.10.008

24. Church TR, Wandell M, Lofton-Day C, Mongin SJ, Burger M, Payne SR, et al. Prospective evaluation of methylated SEPT9 in plasma for detection of asymptomatic colorectal cancer. Gut (2013) 63(2):317-25. doi:10.1136/ gutjnl-2012-304149

25. Wang Z, Chen J, He Q, Al E. MS-HRM detecting free methylation SEPT9 in early diagnosis of colorectal cancer. Guangdong Med J (2012) 33:1732-4. (in Chinese).

26. Su XL, Wang YF, Li SJ, Zhang F, Cui HW. High methylation of the SEPT9 gene in Chinese colorectal cancer patients. Genet Mol Res (2014) 13(2): 2513-20. doi:10.4238/2014.January.17.5

27. Yu D, Zhang X, Lu X. Study on diagnostic value of SEPT9 gene methylation in serum for colorectal cancer. Chin J Clin Lab Sci (2015) 33(9):687-9. doi:10.13602/j.cnki.jcls.2015.09.12

28. Kang J, Jin P, Yang L, Al E. The study of methylation SEPT9 in peripheral DNA for colorectal cancer screening. Natl Med J Chin (2014) 94:3839-41. (in Chinese).

29. Edge SB, Compton CC. The American Joint Committee on Cancer: the 7th edition of the AJCC cancer staging manual and the future of TNM. Ann Surg Oncol (2010) 17(6):1471-4. doi:10.1245/s10434-010-0985-4

30. Devereux TR, Horikawa I, Anna CH, Annab LA, Afshari CA, Barrett JC. DNA methylation analysis of the promoter region of the human telomerase reverse transcriptase (hTERT) gene. Cancer Res (1999) 59(24):6087.

31. Farahzadi R, Fathi E, Mesbah-Namin SA, Zarghami N. Zinc sulfate contributes to promote telomere length extension via increasing telomerase gene expression, telomerase activity and change in the TERT gene promoter CpG island methylation status of human adipose-derived mesenchymal stem cells. PLoS One (2017) 12(11):e188052. doi:10.1371/journal.pone.0188052

32. Tóth K, Galamb O, Spisák S, Wichmann B, Sipos F, Valcz G, et al. The influence of methylated septin9 gene on RNA and protein level in colorectal cancer. Pathol Oncol Res (2011) 17(3):503-9. doi:10.1007/s12253-010-9338-7

33. Grutzmann R, Molnar B, Pilarsky C, Habermann JK, Schlag PM, Saeger HD, et al. Sensitive detection of colorectal cancer in peripheral blood by septin 9 DNA methylation assay. PLoS One (2008) 3(11):e3759. doi:10.1371/journal. pone. 0003759

34. Tóth K, Wasserkort R, Sipos F, Kalmár A, Wichmann B, Leiszter K, et al. Detection of methylated Septin9 in tissue and plasma of colorectal patients with neoplasia and the relationship to the amount of circulating cell-free DNA. PLoS One (2014) 9(12):e115415. doi:10.1371/journal.pone.0115415
35. Potter NT, Hurban P, White MN, Whitlock KD, Lofton-Day CE, Tetzner R, et al. Validation of a real-time PCR-based qualitative assay for the detection of methylated SEPT9 DNA in human plasma. Clin Chem (2014) 60(9):1183-91. doi:10.1373/clinchem.2013.221044

36. Li L, Choi J, Lee K, Sung H, Park SK, Oze I, et al. DNA methylation in peripheral blood: a potential biomarker for cancer molecular epidemiology. J Epidemiol (2012) 22(5):384-94. doi:10.2188/jea.JE20120003

37. Li S, Liu Y, Wang J, Cao C, Yan Y, Yuan P, et al. Application of determination of methylation gene Septin9 in peripheral blood in the screening of colorectal carcinoma. Chin J Gen Surg (2015) 24(12):1756-60. doi:10.3978/j. issn.1005-6947.2015.12.023

38. Jin P, Kang Q, Wang X, Yang L, Yu Y, Li N, et al. Performance of a second-generation methylated SEPT9 test in detecting colorectal neoplasm. J Gastroenterol Hepatol (2015) 30(5):830-3. doi:10.1111/jgh.12855

39. Ørntoft MW, Nielsen HJ, Ørntoft TF, Andersen CL; Danish Study Group on Early Detection of Colorectal Cancer. Performance of the colorectal cancer screening marker Sept9 is influenced by age, diabetes and arthritis: a nested case-control study. BMC Cancer (2015) 15(1):819. doi:10.1186/s12885-015$1832-6$

40. Johnson DA, Barclay RL, Mergener K, Weiss G, KoNig T, Beck J, et al. Plasma Septin9 versus fecal immunochemical testing for colorectal cancer screening: a prospective multicenter study. PLoS One (2014) 9(6):e98238. doi:10.1371/ journal.pone.0098238

41. Wu D, Zhou G, Jin P, Zhu J, Li S, Wu Q, et al. Detection of colorectal cancer using a simplified SEPT9 gene methylation assay is a reliable method for opportunistic screening. J Mol Diagn (2016) 18(4):535-45. doi:10.1016/j. jmoldx.2016.02.005

Conflict of Interest Statement: The authors declare that the research was conducted in the absence of any commercial or financial relationships that could be construed as a potential conflict of interest.

Copyright $\odot 2018$ Xie, Jiang, Li, Sun, Quan, Duan, Li and Chen. This is an openaccess article distributed under the terms of the Creative Commons Attribution License (CC BY). The use, distribution or reproduction in other forums is permitted, provided the original author(s) and the copyright owner are credited and that the original publication in this journal is cited, in accordance with accepted academic practice. No use, distribution or reproduction is permitted which does not comply with these terms. 